\title{
Recognition and management of abdominal compartment syndrome among German anesthetists and surgeons: a national survey
}

Torsten Kaussen ${ }^{1 * \dagger}$, Jens Otto ${ }^{2 \dagger}$, Gerd Steinau ${ }^{2}$, Jörg Höer ${ }^{3}$, Pramod Kadaba Srinivasan ${ }^{4}$, Alexander Schachtrupp ${ }^{2}$

\begin{abstract}
Background: Abdominal compartment syndrome (ACS) is a life threatening condition that may affect any critically ill patient. Little is known about the recognition and management of ACS in Germany.

Methods: A questionnaire was mailed to departments of surgery and anesthesia from German hospitals with more than 450 beds.

Results: Replies (113) were received from 222 eligible hospitals (51\%). Most respondents (95\%) indicated that ACS plays a role in their clinical practice. Intra-abdominal pressure (IAP) is not measured at all by $26 \%$, while it is routinely done by $30 \%$. IAP is mostly (94\%) assessed via the intra-vesical route. Of the respondents, $41 \%$ only measure IAP in patients expected to develop ACS; 64\% states that a simpler, more standardized application of IAP measurement would lead to increased use in daily clinical practice.

Conclusions: German anesthesiologists and surgeons are familiar with ACS. However, approximately one fourth never measures IAP, and there is considerable uncertainty regarding which patients are at risk as well as how often IAP should be measured in them.
\end{abstract}

\section{Introduction}

Abdominal compartment syndrome (ACS) is defined as a persistent intra-abdominal pressure (IAP) of more than $20 \mathrm{mmHg}$ accompanied by new organ dysfunction or failure. Left untreated, this condition has a high mortality rate [1-6]. Intra-abdominal hypertension (IAH) is defined by a sustained or repeated pathological elevation of IAP to more than $12 \mathrm{mmHg}$ and is considered a precursor of ACS [1]. Both IAH and ACS may occur in any patient population requiring intensive care $[7,8]$.

According to surveys in Canada, Great Britain, Australasia, Belgium, China and the USA, detection and management of IAH and ACS are inconsistent $[2,4,9-16]$. Familiarity with the devastating consequences of increased IAP is abundant; however, the relevance of ACS in routine care varies. There is no agreement

\footnotetext{
* Correspondence: Kaussen.Torsten@mh-hannover.de

† Contributed equally

'Department of Pediatric Cardiology and Intensive Care, University Children's Hospital, Hannover Medical School (MHH), OE 6730, Carl-Neuberg St. 1,

30625 Hannover, Germany

Full list of author information is available at the end of the article
}

regarding the indication for IAP measurement and its timing [4]. Moreover, the threshold for decompression is still a matter of debate, as prospective randomized trials are missing $[1,10]$.

Whether a similar level of uncertainty concerning the recognition and management of ACS exists in Germany, and whether this may be related to the techniques available in clinical routine is unknown. We also speculate that a simple, more standardized technique might help improve monitoring of IAP. As comparable studies have yet to be published, we performed this one using a questionnaire.

\section{Methods}

In 2006, a questionnaire (see Additional file 1) was sent to the head physicians of departments of surgery and anesthesia in hospitals with more than 450 beds in Germany. This 450-bed threshold was chosen because hospitals of this size are frequently teaching hospitals and serve as referral centers for smaller hospitals with elective or out-patient surgery.

\section{SpringerOpen ${ }^{\circ}$}

C 2012 Kaussen et al.; licensee Springer This is an open access article distributed under the terms of the Creative Commons Attribution License (http://creativecommons.org/licenses/by/2.0), which permits unrestricted use, distribution, and reproduction in any medium, provided the original work is properly cited. 
According to these criteria, the hospitals were selected via an internet-based hospital registry http://www.krankenhaus.net. A total of 222 questionnaires were sent out. Recipients were asked to reply by fax within 2.5 months. No reminder was sent.

Statistical analysis was calculated using Statistical Package for Social Sciences 12.0.1 for Windows (SPSS Inc., Chicago, IL, USA). Some questions could have more than one answer; in these cases, results were analyzed for multiple responses. The answers were analyzed with respect to training completed by unpaired nonparametric testing (Mann-Whitney $\mathrm{U}$ ).

\section{Results}

A total of 113 questionnaires were returned, four were incomplete or unreadable. Excluding these, 109 questionnaires were analyzed (49\%). Participants stated they had completed training either in anesthesiology (49\%) or surgery (51\%). Their indicated years of clinical practice averaged 21.8 (range 7 to 40 ).

The majority (65\%) stated ACS rarely plays a role in their clinical practice; $24 \%$ are concerned regularly; $6 \%$ often. Not more than $5 \%$ do not encounter this complication. Responding to 'Do you measure IAP?', 28 (26\%) stated 'no'. Of those 81 respondents (73\%) who measured IAP, 48 (59\%) do so 'Only when clinically indicated'. Failure to establish an IAP measurement technique, cited by 22 respondents (28\%, see Figure 1a, b), was the most common reason for not measuring it. The method indicated as most often used for IAP assessment was the measurement of intra-vesical pressure (bladder pressure measurement; $94 \%$, multiple answers possible). In the other cases, a trans-gastric technique was reported. Multiple answers were possible for the question 'In which patients do you measure IAP?'. Respondents most often (41\%) answered that measurement is only performed in patients thought likely to develop ACS (Figure 2).

The majority $(86 \%)$ of respondents stated that the decision to surgically decompress is rather a matter of beginning organ dysfunction than of exceeding pressure thresholds (Figure 3). A simpler, more standardized application would lead to an increased use in 70 of 104 respondents (67\%). Of the 26 participants not measuring IAP, even $77 \%$ think a simplified technique would improve acceptance.

\section{Discussion}

Consensus definitions concerning ACS have been published in order to provide a basis for current treatment $[1,3,8]$. Prospective randomized trials are missing which is probably due to the variable incidence ( $1 \%$ to $15 \%)$, rapid progression and the disease pattern [17-19]. This situation leaves some questions open. Furthermore, the overall purpose of this survey was to study the current status in Germany.

Awareness of ACS and performance of IAP measurements According to our results, ACS plays a role in $95 \%$ of participants' clinical practice. About one third encounter ACS regularly or often. This is comparable to other countries where familiarity with ACS reportedly ranges from $73 \%$ to $99 \%$ of respondents (Table 1). More than one third of respondents from all over the world diagnose at least five cases of ACS each year. Although knowledge regarding ACS seems abundant, about one fourth of respondents claim they never measure IAP. In other surveys, the nonmeasuring rate was mostly comparable (range $2 \%$ to $80 \%$; Table 1). How those participants (who do not measure IAP) establish the diagnosis of ACS remains unclear. Clinical examination of the abdomen has a sensitivity of only $50 \%$ to $60 \%$ which is similar to a coin toss [20-22]. Malbrain et al. demonstrated that also the abdominal perimeter is an inaccurate way for assessing increasing IAP [23].

Among participants measuring IAP, the majority (59\%) stated they perform measurements only if clinically indicated; in contrast, $30 \%$ advocate a routine measurement one to six times per day (Figure 1b). This appears to correlate with respondents tending to perform measurements mostly in patients expected to develop ACS (40\%).

\section{IAP measurement methods}

In accordance with all formerly published surveys, IAP measurement via the bladder is the most frequently used technique also in Germany (Table 1). Of the respondents, $70 \%$ stated that a simpler, more standardized technique would be used more often to assess IAP. This impression is supported by the finding that some respondents refuse bladder pressure measurement because the technique may 'not be established' or appears 'too complex in technical regards'. Both points of criticism appear unjustified. Several studies in humans as well as in animals proved replicability and reliability of the method [24,25]. Further, the measurement techniques have become increasingly simple and user-friendly over the last years, making it no longer possible to speak of an overly complicated IAP measurement technique. For example, the manometer technique, published by Harrahill in 1998 [26] and perfected by Lee [27], offers a maximum simplification of the bladder pressure test and requires no additional instruments other than a ruler and trans-urethral catheter. Using this principle, even commercially available measurement systems have been developed (for example Foleymanometer, Holtech $^{\circledR}$ medical, Charlottenlund, Denmark). Nevertheless, a minimum amount of training for personnel is required to avoid certain pitfalls. This includes, for example, ruling 
(a)

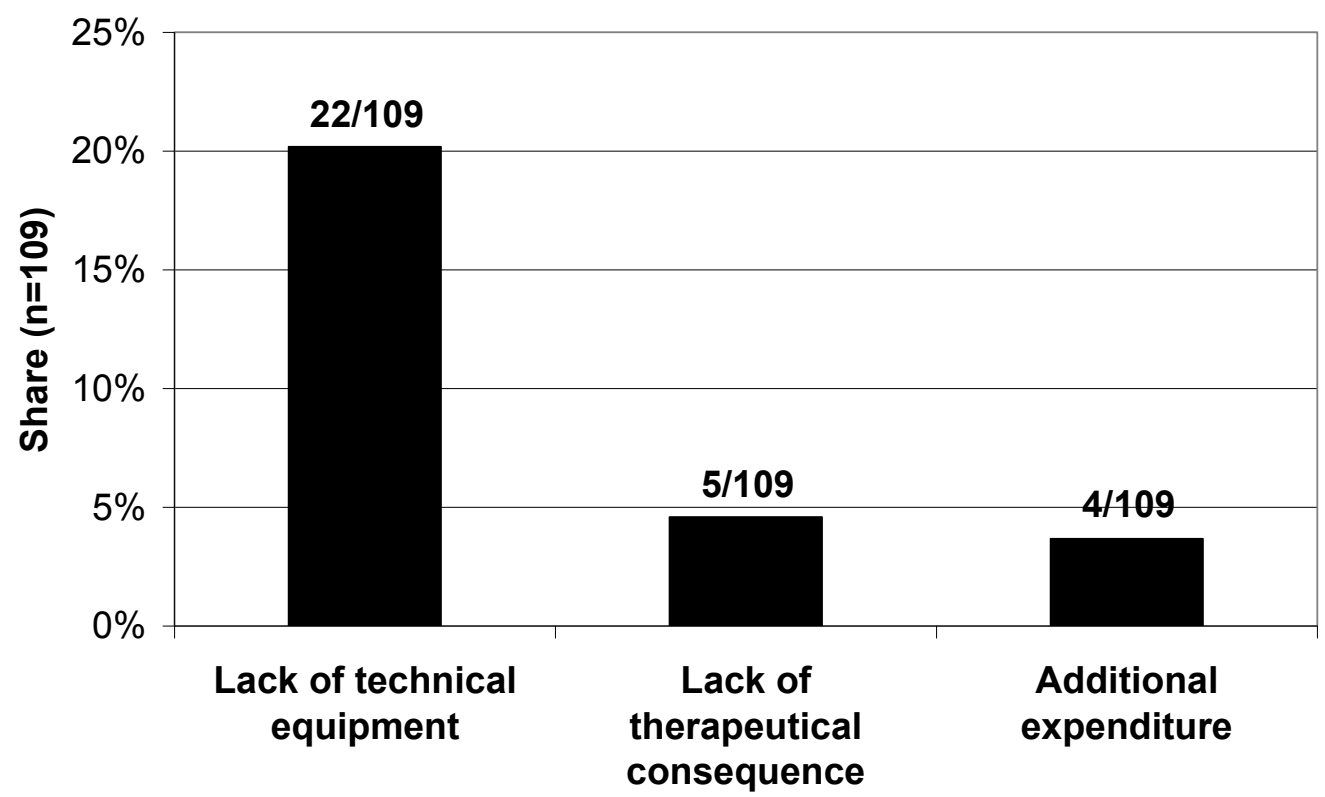

(b)

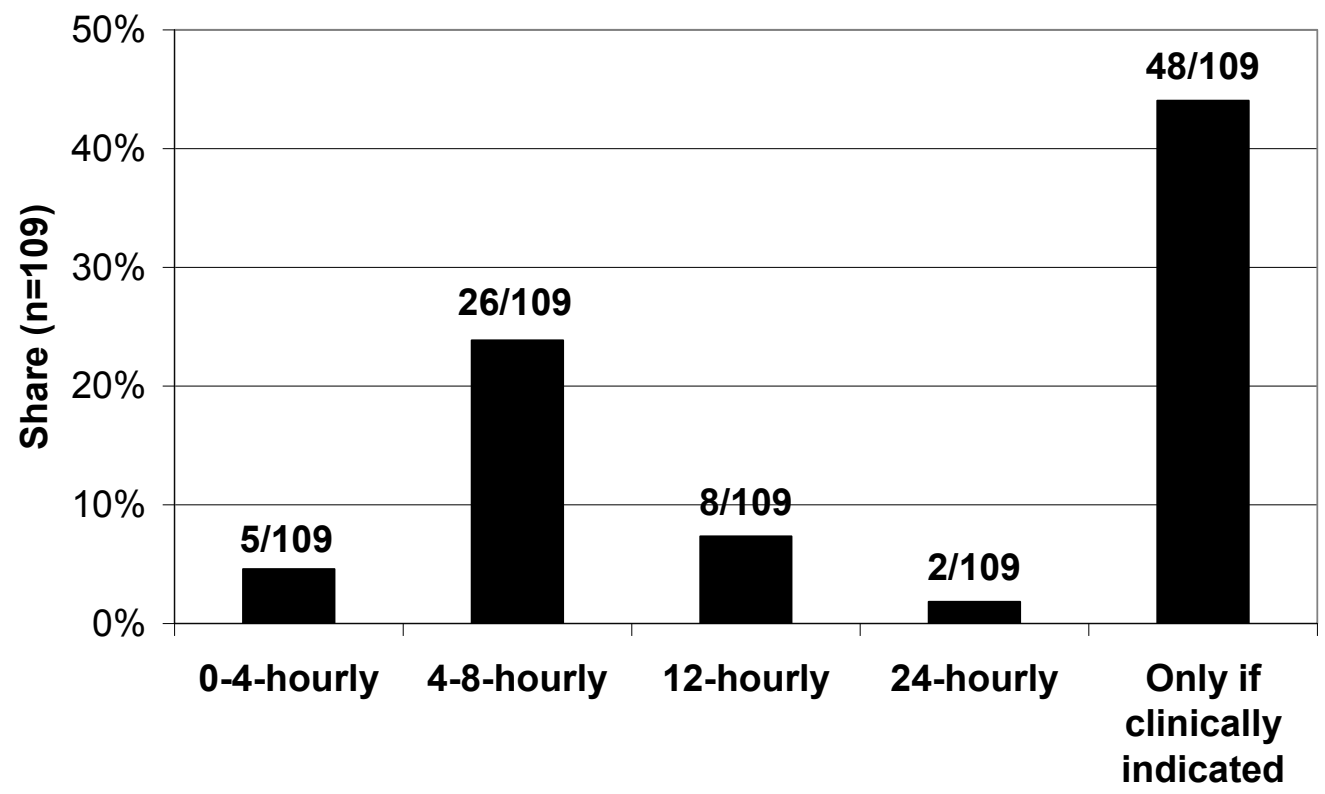

Figure 1 Reasons for not measuring IAP and frequency of IAP measurements. (a) Stated reasons for not measuring IAP. Out of 109 respondents, 28 denied regularly measuring IAP due to the reasons presented (\% of respondents, multiple answers; question 2). (b) Frequency of IAP measurements among those who stated to measure IAP. Of the 109 respondents, 81 elaborated on when to measure IAP (\% of respondents, multiple answers; question 2). 


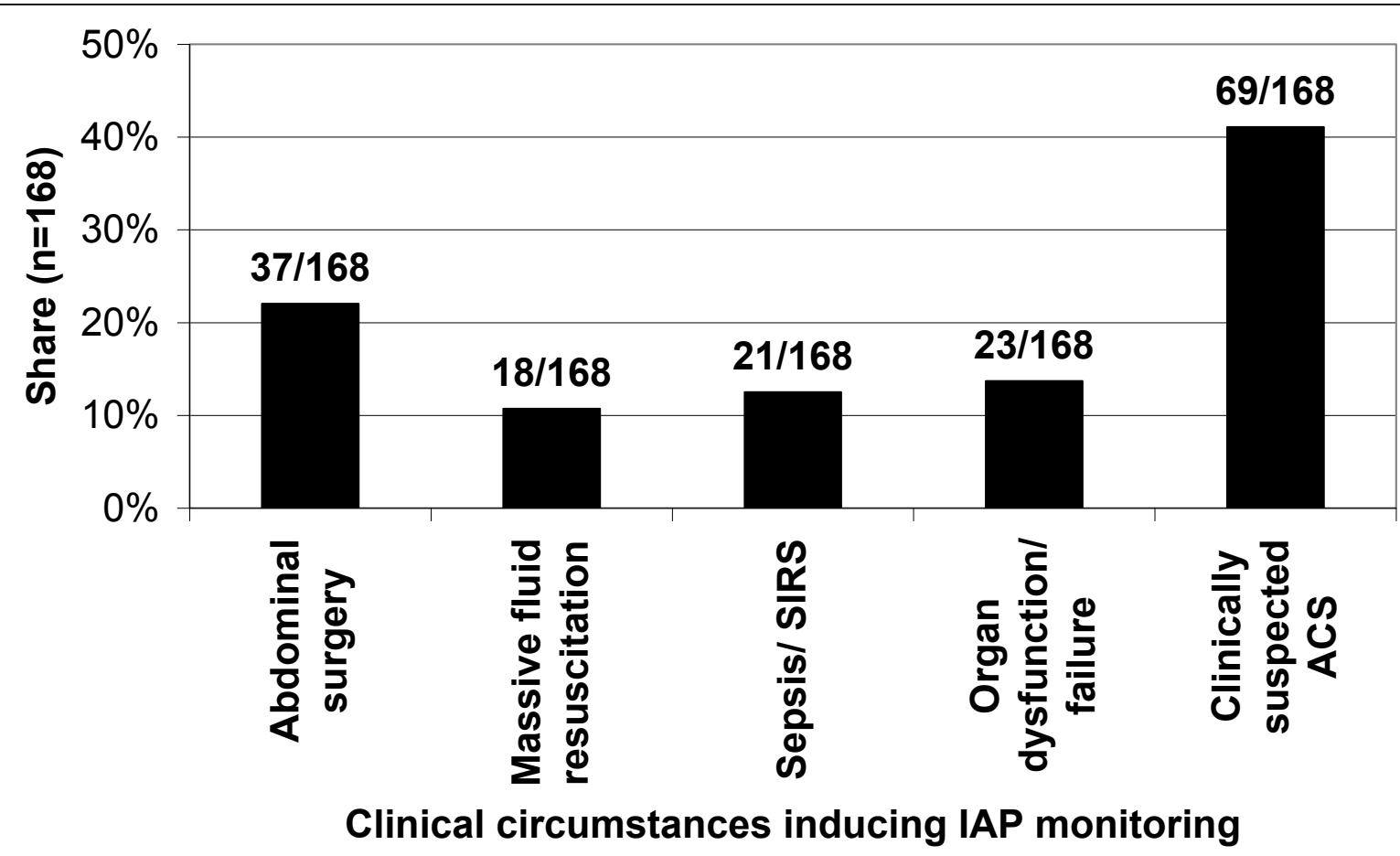

Figure 2 Patient groups which are regularly IAP monitored. Eighty-one stated their criteria regarding in which kind of patients IAP should be measured (\% of respondents, multiple answers; question 4).

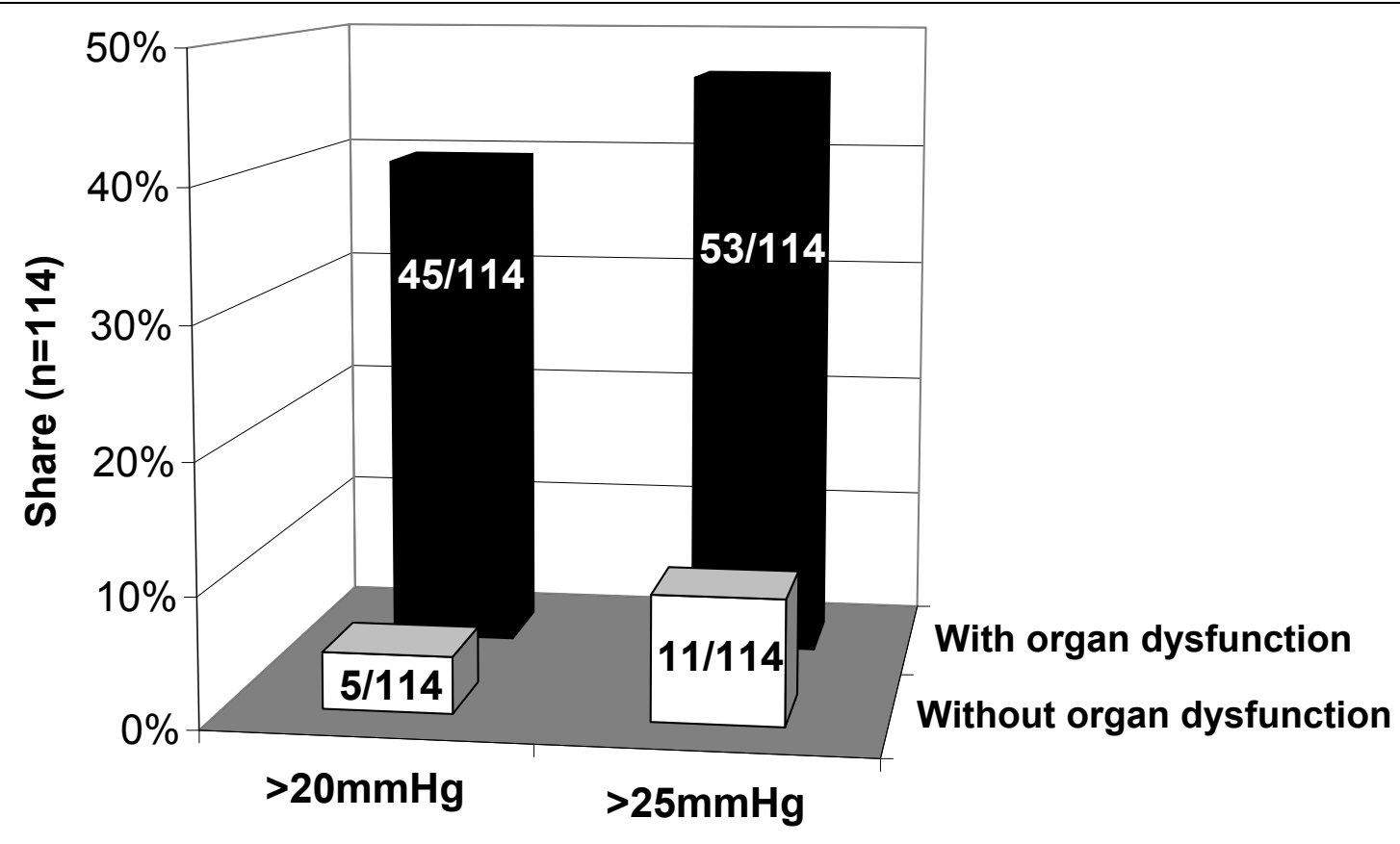

IAP

Figure 3 Critical IAH threshold calling for surgical decompression dependent on organ function and dysfunction. Ninety-four respondents stated their criteria concerning when performing decompressive laparotomy dependend on IAP and organ dysfunction (\% of respondents, multiple answers; question 5) 
Table 1 Comparison between results of current surveys related to IAH and ACS

\begin{tabular}{|c|c|c|c|c|c|c|c|c|c|c|}
\hline Authors & Reference & $\begin{array}{l}\text { Awareness } \\
\text { of ACS }\end{array}$ & $\begin{array}{l}\text { Yearly frequency of AS } \\
\text { at ICUs }\end{array}$ & $\begin{array}{l}\text { Performance of } \\
\text { IAP } \\
\text { measurements }\end{array}$ & $\begin{array}{l}\text { Basis of IAH/ } \\
\text { ACS diagnosis }\end{array}$ & $\begin{array}{l}\text { Measure } \\
\text { method }\end{array}$ & $\begin{array}{l}\text { Frequency of } \\
\text { measurements }\end{array}$ & $\begin{array}{l}\text { Threshold } \\
\text { IAH }\end{array}$ & Threshold ACS & $\begin{array}{l}\text { Experience with/opinion } \\
\text { about } D L \text { and } O A\end{array}$ \\
\hline \multirow[t]{4}{*}{$\begin{array}{l}\text { Mayberry et } \\
\text { al. }\end{array}$} & {$[9]$} & $85 \%$ & 14\%: No cases & $69 \%$ to $95 \%$ & $\begin{array}{l}66 \% \text { IAP } \\
\text { measure }\end{array}$ & IVP & $\begin{array}{l}59 \% \text { If } \\
\text { suspected }\end{array}$ & $\begin{array}{l}15 \mathrm{mmHg} \\
(11 \%)\end{array}$ & & $\begin{array}{l}\text { 86\%: DL if IAH + OD (= } \\
\text { ACS) }\end{array}$ \\
\hline & & & 52\%: One to five cases & & 34\% Clinical & & 6\% Regularly & $\begin{array}{l}18 \mathrm{mmHg} \\
(22 \%)\end{array}$ & & 14\%: DL if IAH alone \\
\hline & & & 33\%: Five cases & & & & & $\begin{array}{l}22 \mathrm{mmHg} \\
(31 \%)\end{array}$ & & $\begin{array}{l}\text { If OA: Bag > absorb. Mesh } \\
>\text { non-absorb. Mesh }\end{array}$ \\
\hline & & & & & & & & $\begin{array}{l}25 \mathrm{mmHg} \\
(12 \%)\end{array}$ & & \\
\hline \multirow[t]{3}{*}{$\begin{array}{l}\text { Kirkpatrick } \\
\text { et al. }\end{array}$} & [10] & $100 \%$ & & $52 \%$ & $\begin{array}{l}43 \% \text { IAP } \\
\text { measure }\end{array}$ & $97 \%$ IVP & & & $25 \mathrm{mmHg}+\mathrm{OD}$ & 8\%: DL if IAH alone \\
\hline & & & & & & $3 \%$ IGP & & & 34 mmHg - OD & $\begin{array}{l}90 \% \text { OA after trauma } \\
\text { surgery }\end{array}$ \\
\hline & & & & & & & & & & $\begin{array}{l}\text { If OA: Bag > VAC > non- } \\
\text { absorb. > absorb. Mesh }\end{array}$ \\
\hline \multirow[t]{7}{*}{$\begin{array}{l}\text { Ravishankar } \\
\text { and Hunter }\end{array}$} & {$[4]$} & $99 \%$ & & $76 \%$ & $\begin{array}{l}76 \% \text { IAP } \\
\text { measure }\end{array}$ & IVP & $\begin{array}{l}93 \% \text { If } \\
\text { suspected }\end{array}$ & & 20 mmHg (29\%) & $\begin{array}{l}\text { 2\%: DL if IAP > } 20 \mathrm{mmHg} \\
\text { alone (= IAH III) }\end{array}$ \\
\hline & & & & & 24\% Clinical & & 4\% After EL & & 25 mmHg (71\%) & $\begin{array}{l}\text { 27\%: DL if IAP }>20 \mathrm{mmHg} \\
+\mathrm{OD}(=\mathrm{ACS})\end{array}$ \\
\hline & & & & & & & $\begin{array}{l}\text { 3\% After EL + } \\
\text { HVR }\end{array}$ & & & $\begin{array}{l}\text { 7\%: DL if IAP > } 25 \mathrm{mmHg} \\
\text { alone (= IAH IV) }\end{array}$ \\
\hline & & & & & & & $\begin{array}{l}\text { 15\%: Zero to } \\
\text { four hourly }\end{array}$ & & & $\begin{array}{l}\text { 64\%: DL if IAP }>25 \mathrm{mmHg} \\
+\mathrm{OD}(=\mathrm{ACS})\end{array}$ \\
\hline & & & & & & & $\begin{array}{l}\text { 27\%: Four to } \\
\text { eight hourly }\end{array}$ & & & \\
\hline & & & & & & & 11\%: 12 hourly & & & \\
\hline & & & & & & & 3\%: 24 hourly & & & \\
\hline \multirow[t]{4}{*}{$\begin{array}{l}\text { Nagappan } \\
\text { et al. }\end{array}$} & [14] & $92 \%$ & $\begin{array}{l}\text { 'Depending on used } \\
\text { thresholds'; ICU- } \\
\text { dependent }\end{array}$ & $48 \%$ to $93 \%$ & 8\% Clinical & $89 \%$ IVP & $8 \%$ Never & $\begin{array}{l}12 \mathrm{mmHg} \\
(11 \%)\end{array}$ & $\mathrm{IAH}+\mathrm{OD}(69 \%)$ & $\begin{array}{l}\text { 92\%: ACS = } \\
\text { decompression (ever) }\end{array}$ \\
\hline & & & & & & $\begin{array}{l}39 \% \\
\text { Direct }\end{array}$ & 53\% Rarely & $\begin{array}{l}20 \mathrm{mmHg} \\
(64 \%)\end{array}$ & $\begin{array}{l}\geq 30 \mathrm{mmHg}-\mathrm{OD} \\
(33 \%)\end{array}$ & $\begin{array}{l}\text { 64\%: 'ACS should be } \\
\text { treated regardless of IAH' }\end{array}$ \\
\hline & & & & & & $6 \%$ IGP & 19\% Regularly & & & \\
\hline & & & & & & $6 \% \operatorname{IRP}$ & $25 \%$ Often & & & \\
\hline \multirow[t]{3}{*}{ Tiwari et al. } & {$[2]$} & $73 \%$ to $97 \%$ & & & $\begin{array}{l}74 \% \text { to } 94 \% \\
\text { IAP measure }\end{array}$ & $\begin{array}{l}90 \% \text { to } \\
96 \% \text { IVP }\end{array}$ & & & $\begin{array}{l}11 \text { to } 30 \mathrm{mmHg} \\
\text { (teaching hospit.) }\end{array}$ & $\begin{array}{l}42 \% \text { Performed DL in } 0 \% \\
\text { to } 25 \% \text { of ACS patients }\end{array}$ \\
\hline & & & & & $\begin{array}{l}60 \% \text { to } 77 \% \\
\text { Clinical }\end{array}$ & $\begin{array}{l}4 \% \text { to } \\
10 \% \\
\text { Direct }\end{array}$ & & & $\begin{array}{l}11 \text { to } 50 \mathrm{mmHg} \\
\text { (district hospital) }\end{array}$ & $\begin{array}{l}19 \% \text { Performed DL in } 25 \% \\
\text { to } 50 \% \text { of ACS patients }\end{array}$ \\
\hline & & & & & $\begin{array}{l}3 \% \text { to } 12 \% \mathrm{CT} \\
\text { scan }\end{array}$ & & & & & $\begin{array}{l}16 \% \text { Performed DL in } 50 \% \\
\text { to } 75 \% \text { of ACS patients }\end{array}$ \\
\hline
\end{tabular}


Table 1 Comparison between results of current surveys related to IAH and ACS (Continued)

\begin{tabular}{|c|c|c|c|c|c|c|c|c|c|c|}
\hline & & & & & $\begin{array}{l}3 \% \mathrm{pH} \\
\text { manometry }\end{array}$ & & & & & $\begin{array}{l}23 \% \text { Performed DL in } 75 \% \\
\text { to } 100 \% \text { of ACS patients }\end{array}$ \\
\hline \multirow[t]{5}{*}{$\begin{array}{l}\text { Kimball et } \\
\text { al. }\end{array}$} & [15] & $75 \%$ to $98 \%$ & 17\%: No cases & $76 \%$ to $98 \%$ & $\begin{array}{l}70 \% \text { IAP + } \\
\text { clinical }\end{array}$ & IVP & $47 \%$ Seldom & $\begin{array}{l}\text { 'Patient } \\
\text { dependent' }\end{array}$ & $\begin{array}{l}20 \text { to } 27 \mathrm{mmHg} \\
(42 \%)\end{array}$ & $\begin{array}{l}\text { 'Useful invasive therapy } \\
\text { options': }\end{array}$ \\
\hline & & & 39\%: One to three cases & & 20\% Clinical & & $23 \%$ Often & & $\begin{array}{l}12 \text { to } 19 \mathrm{mmHg} \\
(18 \% \text { to } 25 \%)\end{array}$ & $\begin{array}{l}\text {-Decompressive } \\
\text { laparotomy }\end{array}$ \\
\hline & & & 27\%: Four to seven cases & & $\begin{array}{l}7 \% \text { IAP } \\
\text { measure }\end{array}$ & & $8 \%$ Routinely & & $\begin{array}{l}12 \text { to } 19 \mathrm{mmHg} \\
(18 \% \text { to } 25 \%)\end{array}$ & -Paracentesis/drains \\
\hline & & & 10\%: Eight to 10 cases & & $3 \%$ Others & & $1 \%$ Other & & & -Escharatomy/fasciotomy \\
\hline & & & 8\%: > Ten cases & & & & & & & $\begin{array}{l}\text {-Peritoneal dialysis } \\
\text { (catheter) }\end{array}$ \\
\hline \multirow[t]{3}{*}{$\begin{array}{l}\text { De Laet et } \\
\text { al. }\end{array}$} & [12] & $80 \%$ & & $41 \%$ & $\begin{array}{l}51 \% \text { IAP } \\
\text { measure }\end{array}$ & $\begin{array}{l}\text { 'Majority' } \\
\text { IVP }\end{array}$ & 59\% Never & $\begin{array}{l}15 \mathrm{mmHg} \\
\text { (IQR } 12 \text { to } \\
15)\end{array}$ & $\begin{array}{l}20 \mathrm{mmHg}(\mathrm{IQR} \\
20 \text { to } 20)\end{array}$ & $\begin{array}{l}\text { 75\% Performed at least } \\
\text { one DL }\end{array}$ \\
\hline & & & & & 49\% Clinical & & $\begin{array}{l}28 \% \text { If } \\
\text { suspected }\end{array}$ & & & $\begin{array}{l}60 \% \text { Performed at least } \\
\text { one OA }\end{array}$ \\
\hline & & & & & & & $\begin{array}{l}12 \% \\
\text { Continuously }\end{array}$ & & & $\begin{array}{l}\text { If OA: Bag > abs. > VAC > } \\
\text { gauze > non-absorb. }\end{array}$ \\
\hline \multirow[t]{4}{*}{ Ejike et al. } & [13] & & & $76 \%$ & $\begin{array}{l}76 \% \text { IAP } \\
\text { measure }\end{array}$ & $68 \%$ IVP & $27 \%$ Never & & & \\
\hline & & & & & 24\% Clinical & $\begin{array}{l}13 \% \\
\text { Direct }\end{array}$ & & & & \\
\hline & & & & & & $\begin{array}{l}+/- \\
\text { Doppler }\end{array}$ & & & & \\
\hline & & & & & & $+/-$ IGP & & & & \\
\hline \multirow[t]{5}{*}{ Zhou et al. } & [16] & & 0\%: No cases & $69 \%$ & 31\% Clinical & $100 \%$ IVP & $\begin{array}{l}88 \% \text { If } \\
\text { suspected }\end{array}$ & & $25 \mathrm{mmHg}$ & $\begin{array}{l}\text { 68\%: First-line therapy } \\
\text { paracentesis }\end{array}$ \\
\hline & & & $44 \%$ : One to three cases & & & $7 \%$ CVP & $71 \%$ Seldom & & & $\begin{array}{l}\text { 56\%: DL if IAP > } 25 \mathrm{mmHg} \\
+\mathrm{OD}(=\mathrm{ACS})\end{array}$ \\
\hline & & & $16 \%:$ Four to seven cases & & & & 29\% Regularly & & & \\
\hline & & & 8\%: Eight to ten cases & & & & 8\% After EL & & & \\
\hline & & & 32\%: > Ten cases & & & & 4\% After HVR & & & \\
\hline \multirow[t]{5}{*}{$\begin{array}{l}\text { Kaussen et } \\
\text { al }^{a}\end{array}$} & & $95 \%$ & 6\%: Never & $75 \%$ & 26\% Clinical & $94 \%$ IVP & $\begin{array}{l}40 \% \text { If } \\
\text { suspected }\end{array}$ & & 20 mmHg (43\%) & $\begin{array}{l}\text { 4\%: DL if IAP > } 20 \mathrm{mmHg} \\
\text { alone (= IAH III) }\end{array}$ \\
\hline & & & 64\%: Seldom & & & $6 \%$ IGP & $\begin{array}{l}\text { 4\%: Zero to } \\
\text { four hourly }\end{array}$ & & 25 mmHg (57\%) & $\begin{array}{l}\text { 39\%: DL if IAP > } 20 \mathrm{mmHg} \\
+\mathrm{OD}(=\mathrm{ACS})\end{array}$ \\
\hline & & & 24\%: Regularly & & & & $\begin{array}{l}22 \% \text { : Four to } \\
\text { eight hourly }\end{array}$ & & & $\begin{array}{l}\text { 10\%: DL if IAP > } 25 \mathrm{mmHg} \\
\text { alone (= IAH IV) }\end{array}$ \\
\hline & & & 6\%: Often & & & & 7\%: 12 hourly & & & $\begin{array}{l}\text { 46\%: DL if IAP > } 25 \mathrm{mmHg} \\
+\mathrm{OD}(=\mathrm{ACS})\end{array}$ \\
\hline & & & & & & & 2\%: 24 hourly & & & \\
\hline $\begin{array}{c}\text { Malbrain et } \\
\text { al. }\end{array}$ & {$[11]$} & $99 \%$ & $0.3 \%$ : No cases & $86 \%$ & $\begin{array}{l}69 \% \text { IAP + } \\
\text { clinical }\end{array}$ & $92 \%$ IVP & $\begin{array}{l}42 \% \text { If } \\
\text { suspected }\end{array}$ & $\begin{array}{l}5 \mathrm{mmHg}(< \\
1 \%)\end{array}$ & $20 \mathrm{mmHg}(27 \%)$ & 74\%: DL if IAH + OD \\
\hline
\end{tabular}


Table 1 Comparison between results of current surveys related to IAH and ACS (Continued)

\begin{tabular}{|c|c|c|c|c|c|c|c|c|c|}
\hline & & & & & & $\begin{array}{l}4 \% \\
\text { Continuously }\end{array}$ & & & \\
\hline & & \multicolumn{2}{|l|}{$62 \%$ : One to five cases } & $\begin{array}{l}24 \% \text { IAP } \\
\text { measure }\end{array}$ & 4\% Direct & $\begin{array}{l}32 \% \text { Four } \\
\text { hourly }\end{array}$ & $\begin{array}{l}10 \mathrm{mmHg} \\
(6 \%)\end{array}$ & 25 mmHg (12\%) & $\begin{array}{l}9 \%: D L \text { if severe } O D \text { (even } \\
\text { without } I A H \text { ) }\end{array}$ \\
\hline & & \multicolumn{2}{|l|}{$20 \%$ : Six to ten cases } & $13 \%$ CT scan & $3 \%$ IGP & $\begin{array}{l}26 \% \text { Six to } \\
\text { eight hourly }\end{array}$ & $\begin{array}{l}12 \mathrm{mmHg} \\
(18 \%)\end{array}$ & $\begin{array}{l}>25 \mathrm{mmHg} \\
(58 \%)\end{array}$ & $\begin{array}{l}\text { 6\%: DL dependent on } \\
\text { cause of ACS }\end{array}$ \\
\hline & & \multicolumn{2}{|l|}{ 6\%: 11 to 15 Cases } & $\begin{array}{l}10 \% \text { Abdom. } \\
\text { perimeter }\end{array}$ & & 6\% 12 hourly & $\begin{array}{l}15 \mathrm{mmHg} \\
(25 \%)\end{array}$ & & $\begin{array}{l}\text { If OA: VAC }(39 \%)>\text { Bag } \\
(24 \%)>\text { mesh }(21 \%)\end{array}$ \\
\hline & & \multicolumn{2}{|l|}{ 5\%: 16 to 20 cases } & $\begin{array}{l}8 \% \text { Abdom. } \\
\text { ultrasound }\end{array}$ & & $2 \% 24$ hourly & $\begin{array}{l}20 \mathrm{mmHg} \\
(29 \%)\end{array}$ & & \\
\hline & & \multirow{3}{*}{\multicolumn{2}{|c|}{ 6\%: > 25 Cases }} & & & & $\begin{array}{l}25 \mathrm{mmHg} \\
(5 \%)\end{array}$ & & \\
\hline & & & & & & & $\begin{array}{l}>25 \mathrm{mHg} \\
(15 \%)\end{array}$ & & \\
\hline & & & & & & & Others (2\%) & & \\
\hline \multirow[t]{3}{*}{$\begin{array}{l}\text { Newcombe [38] } \\
\text { et al. }\end{array}$} & \multirow[t]{3}{*}{$88 \%$} & & \multirow[t]{3}{*}{$92 \%$} & $\begin{array}{l}83 \% \text { IAP } \\
\text { measure }\end{array}$ & $93 \%$ IVP & $21 \%$ Regularly & & $\leq 15 \mathrm{mmHg}(11 \%)$ & \\
\hline & & & & $\begin{array}{l}8 \% \text { IAP + } \\
\text { clinical }\end{array}$ & 7\% Direct & 54\% Sometimes & & $\leq 25 \mathrm{mmHg}(59 \%)$ & \\
\hline & & & & 7\% Clinical & $0 \%$ IGP & 19\% Never & & $\begin{array}{l}>25 \mathrm{mmHg} \\
(30 \%)\end{array}$ & \\
\hline
\end{tabular}

absorb., absorbable (mesh); abdom., abdominal; ACS, abdominal compartment syndrome; AustAsia, Australia and Asia (Australasia); Bag, 'bowel bag' such as 'Bogota bag'; CVP, central venous pressure measurement direct, intra-abdominal pressure measurement via intra-abdominal placed probes; DL, decompressive laparotomy; EL, emergeny laparotomy; hospit., hospital; HVR, high-volume resuscitation; IAH, intra-abdominal hypertension; IAP, intra-abdominal pressure; ICU, intensive care unit; IGP, intra-gastric pressure measurement; IQR, inter-quartile range; IRP, intra-rectal pressure measurement; IVP, intra-vesical (bladder) pressure measurement; non-absorb., non-absorbable (mesh); OA, open abdomen management; OD, organ dysfunction/failure; VAC, vacuum-assisted. ${ }^{a}$ Unpublished work. 
out a neurogenic or organic bladder dysfunction, ensuring sufficient relaxation of the local abdominal muscles, and the correct steady positioning of the patient with a continuous transparent reference point for the measurement of pressure equivalents.

Other indirect methods such as intra-gastric and intra-rectal pressure measurements rather constitute an exception than the rule and were stated to be performed by no more than $6 \%$ of respondents (Table 1 ). This is noteworthy in so far as different commercially available measurement systems, meanwhile, have been developed which allow to continuously monitor IAP levels via the stomach (for example CiMON ${ }^{\circledR}$, Pulsion ${ }^{\circledR}$ Medical Systems, Munich, Germany or 'IAP catheter', Spiegelberg ${ }^{\circledR}$, Hamburg, Germany). Continuous measurement systems are able to minimize health care providers' workload as well as ensure non-stop observation of especially at risk patients. Pressure transducers, which are directly inserted into the abdomen, even more precisely reflect the IAP. Further information with respect to direct and indirect IAP measurement methods, as well as to continuous and intermittent techniques can be found on excellent reviews which have been published by Malbrain [28] and De Keulenaer [29].

If various measurement procedures are available, the illness and the dynamic of possibly increasing abdominal pressure should be considered. The higher the IAP, and respectively, the more quickly it is increasing, the sooner continuous pressure monitoring should be considered in order to begin the necessary therapeutic procedures, including invasive ones, in time. Apparently, it is of utmost importance that IAP be quantified when, as recommended by the World Society on the Abdominal Compartment Syndrome (WSACS), certain risk factors are present (Figure 2; Table 2). Using appropriate therapy algorithms, it should thereby become possible to react earlier and assertively enough to IAH that an ACS case does not even arise.

\section{IAP thresholds}

Although the WSACS published definitions more than 5 years ago [3], there is still a remarkable lack of knowledge concerning the recommended threshold values in relation to IAH and ACS (Table 1). On the one hand, this might be caused by a lack of awareness of current literature; on the other, this might be influenced by personal experience, which might differ from published results and consensus. While the values gathered in the course of the surveys were partially over the WSACS limits for adults, the majority of pediatricians reported much lower values. This reflects the clinical impression that IAH and ACS can appear at much lower levels of abdominal pressure in children. In the framework of the 5th WSACS World Congress 2011 and using the data available at that time, Ejike et al. correctly demanded the establishment of pediatric limits (IAH: IAP > $10 \mathrm{mmHg}$ ACS: IAH + new organ dysfunction) (KT et al., unpublished work) [30].

\section{Surgical therapy options}

Most of our respondents decide to decompress the abdomen based on the presence of organ dysfunction or failure in combination with IAH (Figure 3). The attitude towards the critical threshold ( $>20 \mathrm{mmHg}$ or $>25$ $\mathrm{mmHg}$ ) divides respondents into two groups of similar size (39\% vs $46 \%$ ). This is comparable to the surveys done by Ravishankar and Mayberry ([4,9], Table 1). One reason may be the lack of evidence as prospective outcome studies are missing and the mortality rate of ACS has remained high despite decompression [18,31]. Tiwari describes a reluctance among surgeons to operate patients with ACS [2]. They probably try to avoid complications associated with decompression and the management of an open abdomen as described by Kirkpatrick et al. in their survey of Canadian surgeons [10]. This restraint might arise from reports about sudden deaths following surgical decompression in patients suffering from IAH and ACS [32-34]. Fatal outcome in these patients might be related to fatal pulmonary embolism caused by venous stasis in the splanchnic venous capacitance pool during IAH/ACS. It has also been stated that lethal acute circulatory collapses and asystolia after decompression might be caused by the release of anaerobic metabolic products and inflammatory mediators from prior less perfused tissues (ischemia-reperfusion syndrome $[35,36])$. This pathogenesis, however, is not generally accepted.

Cheatham and Safcsak have demonstrated that routinely monitoring adult patients at risk and a stage-by-stageguided therapy algorithm comprising medical as well as surgical options may considerably reduce patient mortality by up to 50\% [37]. This also supports not delaying decompression when necessary. Respondents as well as participants in other surveys are familiar with decompressive laparotomy and more or less perform this escalated therapy option partly in combination with open-abdomen management often (Table 1). In this connection, it should be noted that, in all studies, the majority of physicians interviewed work in tertiary care hospitals and high-level ICUs. To a lesser degree, these results reflect circumstances found in basic and regular care hospitals where recognition and standardized therapy of IAH and ACS seem to lead a miserable existence.

\section{Limitations}

Surveys are known to have limitations as results represent personal assessment rather than objective data. A limitation might be that the survey was only sent to the heads of departments and not to section members. It can 
Table 2 Risk factors for IAH/ACS as proposed by the WSACS (adapted from [24])

\begin{tabular}{|c|c|}
\hline Category & Risk factors \\
\hline \multirow{11}{*}{$\begin{array}{l}\text { 1. Diminished abdominal wall } \\
\text { compliance }\end{array}$} & Mechanical ventilation, especially fighting with the ventilator and use of accessory respiratory muscles \\
\hline & Use of positive end expiratory pressure (PEEP) or the presence of auto-PEEP \\
\hline & Basal pleuropneumonia \\
\hline & High body mass index \\
\hline & Pneumoperitoneum \\
\hline & Abdominal (vascular) surgery, especially with tight abdominal closures \\
\hline & Pneumatic anti-shock garments \\
\hline & Prone and other body positioning \\
\hline & Abdominal wall bleeding or rectus sheath hematomas \\
\hline & Correction of large hernias, gastroschisis or omphalocele \\
\hline & Burns with abdominal eschars \\
\hline \multirow{3}{*}{$\begin{array}{l}\text { 2. Increased intra-luminal } \\
\text { contents }\end{array}$} & Gastroparesis/gastric distension/ileus/colonic pseudo-obstruction \\
\hline & Abdominal tumor \\
\hline & Retroperitoneal/abdominal wall hematoma \\
\hline \multirow{4}{*}{$\begin{array}{l}\text { 3. Increased intra-abdominal } \\
\text { contents }\end{array}$} & Liver dysfunction with ascites \\
\hline & Abdominal infection (pancreatitis, peritonitis, abscess, etc.) \\
\hline & Hemoperitoneum/pneumoperitoneum \\
\hline & Acidosis ( $\mathrm{pH}$ below 7.2) \\
\hline \multirow[t]{7}{*}{ 4. Capillary leak } & Hypothermia (core temperature below $33^{\circ} \mathrm{C}$ ) \\
\hline & Polytransfusion/trauma (> 10 units of packed red cells/24 h \\
\hline & $\begin{array}{l}\text { Coagulopathy (platelet count below } 5,000 / \mathrm{mm}^{3} \text {, an activated partial thromboplastin time (aPTT) more than } 2 \text { times } \\
\text { normal, a prothrombin time (PTT) below } 50 \% \text {, or an international standardized ration (INR) more than 1.5) }\end{array}$ \\
\hline & Sepsis (as defined by the American-European Consensus Conference definitions) \\
\hline & Bacteremia \\
\hline & Massive fluid resuscitation (> 5 I of colloid or crystalloid/24 h with capillary leak and positive fluid balance) \\
\hline & Major burns \\
\hline
\end{tabular}

be argued that the majority of head physicians carry out more administrative than clinical-curative tasks; meaning, they may not be sufficiently informed about current developments in the treatment of IAH and ACS which could have had a negative impact on the validity of the survey results. On the other hand, it appears less likely that establishment of IAP measurements nor therapeutic procedures, including decompressive laparotomies, are carried out in a department without the decision of the head of the department to do so. As a result, head physicians, even if less involved in everyday clinical work, are considered to be sufficiently knowledgeable to answer the questions posed.

A further limitation is that participants might have simply used their gut feeling instead of clinical databanks to answer the questions. Since doing so would cause more work, it must be assumed that the response rate would have been worse (range of response rates of published IAP surveys: $6 \%$ to $90 \%$; Table 3 ). Therefore, it was decided not to perform a databank survey. The results, which are, to a great extent, identical to the available literature, appear not to express an undue bias (Table 1).
It was decided to send questionnaires to intensive care units of surgical and anesthesiological departments. Due to the current structure in Germany, patients with IAH/ ACS are predominantly placed in departments of surgery and anesthesiology and by far less often present in internal departments.

However, the data display an attitude towards the management of ACS in Germany, thereby, demonstrating a lack of consensus and certainty. This might help guide future studies with a multi-center prospective randomized approach.

\section{Conclusion}

ACS is known among German anesthesiologists and surgeons, and both groups do not differ in their attitude towards this complication. Measurement of bladder pressure appears to be the current standard to assess IAP. However, about one fourth of responding physicians in Germany never measure IAP, and there is considerable uncertainty about which patients are at risk of developing ACS and how often IAP should be measured. Regarding the IAP threshold for decompression 
Table 3 Overview and structural description of current surveys related to IAH and ACS

\begin{tabular}{|c|c|c|c|c|c|c|c|c|}
\hline Authors & Reference & Country & Year $^{\mathrm{a}}$ & $\begin{array}{l}\text { Questionnaires } \\
\text { (returned/sent) }\end{array}$ & $\begin{array}{l}\text { Response } \\
\text { rate }\end{array}$ & Communication channel & Specialty of participitants & Level of medical care \\
\hline Mayberry et al. & [9] & USA & $\begin{array}{c}1999 / \\
1997\end{array}$ & 292/473 & $62 \%$ & Mail & Trauma surgeons & 85\% Teaching hospitals \\
\hline Kirkpatrick et al. & {$[10]$} & Canada & $\begin{array}{l}2005 / \\
2005\end{array}$ & $86 / 102$ & $84 \%$ & Mail and email & Trauma surgeons & \\
\hline $\begin{array}{l}\text { Ravishankar and } \\
\text { Hunter }\end{array}$ & [4] & UK & 2005/NA & 137/207 & $66 \%$ & Mail & Intensivists & \\
\hline \multirow[t]{3}{*}{ Nagappan et al. } & [14] & Australasia & $\begin{array}{l}2005 / \\
2004\end{array}$ & $36 / 40$ & $90 \%$ & Hand-out at workshop & ICU registrars & 72\% High-level ICU \\
\hline & & & & & & & & 10\% Medium-level ICU \\
\hline & & & & & & & & 3\% Low-level ICU \\
\hline \multirow[t]{2}{*}{ Tiwari et al. } & {$[2]$} & UK & $\begin{array}{l}2006 / \\
2004\end{array}$ & $127 / 222$ & $57 \%$ & Mail & Intensivists & 25\% Teaching hospitals \\
\hline & & & & & & & & 75\% District hospitals \\
\hline \multirow[t]{5}{*}{ Kimball et al. } & [15] & USA & $\begin{array}{l}2006 / \\
2001\end{array}$ & $1622 / 4538$ & $36 \%$ & Mail & $35 \%$ Surgeons & \\
\hline & & & & & & & $32 \%$ Internists & \\
\hline & & & & & & & 18\% Pediatricians & \\
\hline & & & & & & & 10\% Anesthetics & \\
\hline & & & & & & & 1\% Emergency doctors & \\
\hline \multirow[t]{2}{*}{ De Laet et al. } & [12] & Belgium & $\begin{array}{l}2007 / \\
2005\end{array}$ & $41 / 689$ & $6 \%$ & Email & Surgeons & 73\% Teaching hospitals \\
\hline & & & & & & & & $27 \%$ District hospitals \\
\hline \multirow[t]{5}{*}{ Ejike et al. } & [13] & $\begin{array}{c}60 \% \\
\text { America }\end{array}$ & $\begin{array}{l}2010 / \\
2006\end{array}$ & $517 / 1107$ & $47 \%$ & $\begin{array}{l}\text { Hand-out at pediatric } \\
\text { congresses }\end{array}$ & $60 \%$ Pediatric nurses & $81 \%$ Tertiary care hospitals \\
\hline & & 26\% Europe & & & & & 30\% Pediatric intensivists & 14\% Community hospitals \\
\hline & & $\begin{array}{c}12 \% \\
\text { Australasia }\end{array}$ & & & & & $4 \%$ General pediatricians & 2\% Private practise \\
\hline & & & & & & & $\begin{array}{c}6 \% \text { Other pediatric health care } \\
\text { providers }\end{array}$ & 1\% Clinics \\
\hline & & & & & & & & $2 \%$ Others \\
\hline \multirow[t]{4}{*}{ Zhou et al. } & [16] & China & $\begin{array}{l}2011 / \\
2010\end{array}$ & 108/141 & $77 \%$ & Mail & 39\% Emergency doctor & 100\% Tertiary care hospitals \\
\hline & & & & & & & $36 \%$ Internists & \\
\hline & & & & & & & 19\% Surgeons & \\
\hline & & & & & & & $6 \%$ Anesthetics & \\
\hline \multirow[t]{2}{*}{ Kaussen et al. } & & Germany & $\begin{array}{l}2012^{b} / \\
2006\end{array}$ & $113 / 222$ & $51 \%$ & Mail & $52 \%$ Surgeons & $\begin{array}{c}\text { Larger hospitals with }>450 \\
\text { patient beds }\end{array}$ \\
\hline & & & & & & & 48\% Anesthetics & \\
\hline \multirow[t]{2}{*}{ Malbrain et al. } & [11] & $\begin{array}{c}58 \% \\
\text { America }\end{array}$ & $\begin{array}{l}2012 / \\
2007\end{array}$ & $2244 / 8081$ & $28 \%$ & $\begin{array}{l}\text { Contacting via email/online- } \\
\text { questionnaire }\end{array}$ & 37\% ICU physicians & \\
\hline & & $32 \%$ Europe & & & & & $23 \%$ Surgeons & \\
\hline
\end{tabular}


Table 3 Overview and structural description of current surveys related to IAH and ACS (Continued)

\begin{tabular}{|c|c|c|c|c|c|c|c|}
\hline & & $\begin{array}{c}9 \% \\
\text { Australasia }\end{array}$ & & & & $21 \%$ Anesthetics & \\
\hline & & $1 \%$ Africa & & & & $8 \%$ Internists & \\
\hline & & & & & & $6 \%$ Pediatricians & \\
\hline & & & & & & 2\% Emergency physicians & \\
\hline & & & & & & $1 \%$ Cardiologists & \\
\hline & & & & & & $2 \%$ Others & \\
\hline \multirow[t]{3}{*}{ Newcombe et al. } & [38] & $97 \%$ USA & $\begin{array}{l}2012 / \\
2010\end{array}$ & $433 / 691$ & Hand-out at pediatric congress & Pediatric nurses & $>60 \%$ Tertiary care hospitals \\
\hline & & & & & & & $<30 \%$ Community hospitals \\
\hline & & & & & & & $<10 \%$ Others \\
\hline
\end{tabular}

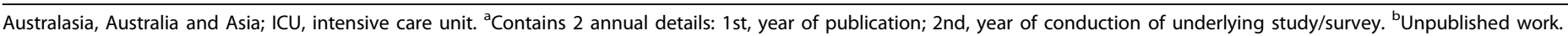


(20 or $25 \mathrm{mmHg}$ ), respondents remain undecided. These findings lead to the overall impression that recognition and management of IAH or ACS need to be further established in Germany.

\section{Additional material}

\section{Additional file 1: Appendix}

\section{Abbreviations}

ACS: abdominal compartment syndrome; IAH: intra-abdominal hypertension; IAP: intra-abdominal pressure; WSACS: World Society on the Abdominal Compartment Syndrome.

\section{Acknowledgements}

The authors would like to thank the directors of the departments of surgery and anesthesia who sent in their replies. We would also like to thank Dawn Nichols for linguistic advice. The charges on the publication of this article were taken on by meas of the promotion programme "Open access publishing" by the German research council (Deutsche Forshungsgemeinschaft).

This article has been published as part of Annals of Intensive Care Volume 2 Supplement 1, 2012: Diagnosis and management of intra-abdominal hypertension and abdominal compartment syndrome. The full contents of the supplement are available online at http://www.annalsofintensivecare. com/supplements/2/S1

\section{Author details}

1Department of Pediatric Cardiology and Intensive Care, University Children's Hospital, Hannover Medical School (MHH), OE 6730, Carl-Neuberg St. 1, 30625 Hannover, Germany. ${ }^{2}$ Department of Surgery, RWTH Aachen University Hospital, Pauwelsstrasse 30, 52074 Aachen, Germany. ${ }^{3}$ Department of Surgery, Hochtaunus-Kliniken Bad Homburg, Urseler Str. 33, 61348- Bad Homburg v. d. Höhe, Germany. ${ }^{4}$ Institute of Experimental Animal Science, RWTH Aachen University Hospital, Pauwelsstrasse 30, 52070 Aachen, Germany.

\section{Authors' contributions}

Literature research was done by JO, TK and AS. Data collection was mainly performed by $\mathrm{JO}$ and AS. The article was written by TK, JO and AS and reviewed by GS, JH and AS. PKS delivered linguistic advice and substantially revised the manuscript. All authors read and approved the final manuscript.

\section{Competing interests}

In addition to his assistant professorship at the Technical University of Aachen (Germany), Alexander Schachtrupp is head of the Department of Medical Sciences at B. Braun Melsungen in Germany. B. Braun does not distribute any medical devices or products concerning the diagnosis and/or treatment of $\mathrm{AH}$ or ACS. The other authors declare that they have no competing interests.

Published: 5 July 2012

\section{References}

1. Malbrain ML, Cheatham ML, Kirkpatrick A, Sugrue M, De Waele J, Ivatury R: Abdominal compartment syndrome: it's time to pay attention! Intensive Care Med 2006, 32:1912-1914.

2. Tiwari A, Myint F, Hamilton G: Recognition and management of abdominal compartment syndrome in the United Kingdom. Intensive Care Med 2006, 32:906-909.

3. Malbrain ML, Cheatham ML, Kirkpatrick A, Sugrue M, Parr M, De Waele J, Balogh Z, Leppaniemi A, Olvera C, Ivatury R, D'Amours S, Wendon J, Hillman K, Johansson K, Kolkman K, Wilmer A: Results from the international conference of experts on intra-abdominal hypertension and abdominal compartment syndrome. I. Definitions. Intensive Care Med 2006, 32:1722-1732.
4. Ravishankar N, Hunter J: Measurement of intra-abdominal pressure in intensive care units in the United Kingdom: a national postal questionnaire study. Br J Anaesth 2005, 94:763-766.

5. Malbrain ML, Chiumello D, Pelosi $P$, Bihari $D$, Innes R, Ranieri VM, del Turco M, Wilmer A, Brienza N, Malcangi V, Cohen J, Japiassu A, De Keulenaer BL, Daelemans R, Jacquet L, Laterre PF, Frank G, de Souza P, Cesana B, Gattinoni L: Incidence and prognosis of intraabdominal hypertension in a mixed population of critically ill patients: a multiplecenter epidemiological study. Crit Care Med 2005, 33:315-322.

6. Malbrain ML, Chiumello D, Pelosi P, Wilmer A, Brienza N, Malcangi V, Bihari D, Innes R, Cohen J, Singer P, Japiassu A, Kurtop E, De Keulenaer BL, Daelemans R, del Turco M, Cosimini P, Ranieri M, Jacquet L, Laterre PF, Gattinoni L: Prevalence of intra-abdominal hypertension in critically ill patients: a multicentre epidemiological study. Intensive Care Med 2004, 30:822-829.

7. Ivatury RR: Abdominal compartment syndrome: a century later, isn't it time to accept and promulgate? Crit Care Med 2006, 34:2494-2495.

8. Cheatham ML, Malbrain ML, Kirkpatrick A, Sugrue M, Parr M, De Waele J, Balogh Z, Leppaniemi A, Olvera C, Ivatury R, D'Amours S, Wendon J, Hillman K, Wilmer A: Results from the international conference of experts on intra-abdominal hypertension and abdominal compartment syndrome. II. Recommendations. Intensive Care Med 2007, 33:951-962

9. Mayberry JC, Goldman RK, Mullins RJ, Brand DM, Crass RA, Trunkey DD: Surveyed opinion of American trauma surgeons on the prevention of the abdominal compartment syndrome. J Trauma 1999, 47:509-513.

10. Kirkpatrick AW, Laupland KB, Karmali S, Bergeron E, Stewart TC, Findlay C, Parry N, Khetarpal S, Evans D: Spill your guts! Perceptions of Trauma Association of Canada member surgeons regarding the open abdomen and the abdominal compartment syndrome. J Trauma 2006, 60:279-286.

11. Kimball EJ, Kim W, Cheatham ML, Malbrain ML: Clinical awareness of intraabdominal hypertension and abdominal compartment syndrome in 2007. Acta Clin Belg Suppl 2007, 1:66-73.

12. De Laet IE, Hoste EA, De Waele JJ: Survey on the perception and management of the abdominal compartment syndrome among Belgian surgeons. Acta Chir Belg 2007, 107:648-652.

13. Ejike JC, Newcombe J, Baerg J, Bahjri K, Mathur M: Understanding of abdominal compartment syndrome among pediatric healthcare providers. Crit Care Res Pract 2010, Epub 876013.

14. Nagappan R, Ernest D, Whitfield A: Recognition and management of intraabdominal hypertension and abdominal compartment syndrome. Crit Care Resusc 2005, 7:298-302.

15. Kimball EJ, Rollins MD, Mone MC, Hansen HJ, Baraghoshi GK, Johnston C, Day ES, Jackson PR, Payne M, Barton RG: Survey of intensive care physicians on the recognition and management of intra-abdominal hypertension and abdominal compartment syndrome. Crit Care Med 2006, 34:2340-2348.

16. Zhou JC, Zhao HC, Pan $\mathrm{KH}, \mathrm{Xu}$ QP: Current recognition and management of intra-abdominal hypertension and abdominal compartment syndrome among tertiary Chinese intensive care physicians. J Zhejiang Univ Sci B 2011, 12:156-162.

17. Balogh $Z$, Jones F, D'Amours S, Parr M, Sugrue M: Continuous intraabdominal pressure measurement technique. Am J Surg 2004, 188:679-684.

18. De Waele JJ, Hoste EA, Malbrain ML: Decompressive laparotomy for abdominal compartment syndrome-a critical analysis. Crit Care 2006, 10: R51.

19. Hong JJ, Cohn SM, Perez JM, Dolich MO, Brown M, McKenney MG: Prospective study of the incidence and outcome of intra-abdominal hypertension and the abdominal compartment syndrome. Br J Surg 2002, 89:591-596

20. Schachtrupp A, Jansen M, Bertram P, Kuhlen R, Schumpelick V: Abdominal compartment syndrome: significance, diagnosis and treatment. Anaesthesist 2006, 55:660-667.

21. Kirkpatrick AW, Brenneman FD, McLean RF, Rapanos T, Boulanger BR: Is clinical examination an accurate indicator of raised intra-abdominal pressure in critically injured patients? Can J Surg 2000, 43:207-211.

22. Sugrue M, Bauman A, Jones F, Bishop G, Flabouris A, Parr M, Stewart A, Hillman K, Deane SA: Clinical examination is an inaccurate predictor of intraabdominal pressure. World J Surg 2002, 26:1428-1431.

23. Malbrain ML, De Laet I, Van RN, Schoonheydt K, Dits H: Can the abdominal perimeter be used as an accurate estimation of intra-abdominal pressure? Crit Care Med 2009, 37:316-319. 
24. De Waele JJ, De Laet I, Malbrain ML: Rational intraabdominal pressure monitoring: how to do it? Acta Clin Belg Supp/ 2007, 1:16-25

25. Balogh Z, De Waele JJ, Malbrain ML: Continuous intra-abdominal pressure monitoring. Acta Clin Belg Suppl 2007, 1:26-32.

26. Harrahill M: Intra-abdominal pressure monitoring. J Emerg Nurs 1998 24:465-466.

27. Lee SL, Anderson JT, Kraut EJ, Wisner DH, Wolfe BM: A simplified approach to the diagnosis of elevated intra-abdominal pressure. J Trauma 2002, 52:1169-1172.

28. Malbrain ML: Different techniques to measure intra-abdominal pressure (IAP): time for a critical re-appraisal. Intensive Care Med 2004, 30:357-371.

29. De Keulenaer BL, Regli A, Malbrain ML: Intra-abdominal measurement techniques: is there anything new? Am Surg 2011, 77(Suppl 1):S17-22

30. Ejike JC, Mathur M, Moores DC: Abdominal compartment syndrome: focus on the children. Am Surg 2011, 77:72-77.

31. De Waele JJ, Pletinckx P, Blot S, Hoste E: Saline volume in transvesical intra-abdominal pressure measurement: enough is enough. Intensive Care Med 2006, 32:455-459.

32. Morris JA Jr, Eddy VA, Blinman TA, Rutherford EJ, Sharp KW: The staged celiotomy for trauma. Issues in unpacking and reconstruction. Ann Surg 1993, 217:576-584.

33. Schein M, Wittmann DH, Aprahamian CC, Condon RE: The abdominal compartment syndrome: the physiological and clinical consequences of elevated intra-abdominal pressure. J Am Coll Surg 1995, 180:745-753.

34. Shelly MP, Robinson AA, Hesford JW, Park GR: Haemodynamic effects following surgical release of increased intra-abdominal pressure. $\mathrm{Br} J$ Anaesth 1987, 59:800-805.

35. Bathe OF, Chow AW, Phang PT: Splanchnic origin of cytokines in a porcine model of mesenteric ischemia-reperfusion. Surgery 1998, 123:79-88

36. Cullen DJ, Coyle JP, Teplick R, Long MC: Cardiovascular, pulmonary, and renal effects of massively increased intra-abdominal pressure in critically ill patients. Crit Care Med 1989, 17:118-121.

37. Cheatham ML, Safcsak K: Is the evolving management of intra-abdominal hypertension and abdominal compartment syndrome improving survival? Crit Care Med 2010, 38:402-407.

38. Newcombe J, Mathur M, Bahjiri K, Ejike JC: Pediatric critical care nurses' experience with abdominal compartment syndrome. Annals of Intensive Care.

doi:10.1186/2110-5820-2-S1-S7

Cite this article as: Kaussen et al:: Recognition and management of abdominal compartment syndrome among German anesthetists and surgeons: a national survey. Annals of Intensive Care 2012 2(Suppl 1):S7.

\section{Submit your manuscript to a SpringerOpen ${ }^{\circ}$ journal and benefit from:}

- Convenient online submission

- Rigorous peer review

- Immediate publication on acceptance

- Open access: articles freely available online

- High visibility within the field

- Retaining the copyright to your article

Submit your next manuscript at $>$ springeropen.com 